\title{
puente pretensado sobre el río TRENT
}

\author{
R. M. FINCH y R. TRAVERS MORGAN and Partners, ingenieros
} JAMES MILLER and Partners, constructores

$562-3$

El nuevo puente de Clifton obedece a la necesidad que hace mucho tiempo se hacia-sentir de un paso complementario sobre el río Trent en las proximidades de Nottingham. La gran densidad del tráfico que debía hacer frente al paso sobre el río, motivando embotellarnientos de vehiculos. ha sido la causa que ha precipitado la realización de este proyecto. El nuevo puente viene a remediar estos inconvenientes. Esta obra forma parte de un plan general de arterias de circulación provistas de dos calzadas; pero la urgencia del paso, ha sido la causa determinante de su realización independientemente del referido proyecto de carreteras.

El puente, de hormigón pretensado, tiene una anchura total de $14 \mathrm{~m}$, de fos que 6,70 son para la calzada; 2,75, para pista de ciclos; 1,80, para un andén, y el resto, para bordillo y separaciones. Cuando el programa de carreteras se desarrolle, a este puente se le adosará una segunda estructura cuyo tablero será simétrico al existente.

El nuevo puente de Clifton, en esviaje, formando un ángulo de $24^{\circ}$ con el eje, se compone de 6 tramos y se halla situado a unos $2.400 \mathrm{~m}$ aguas arriba del puente llamado de Trent.

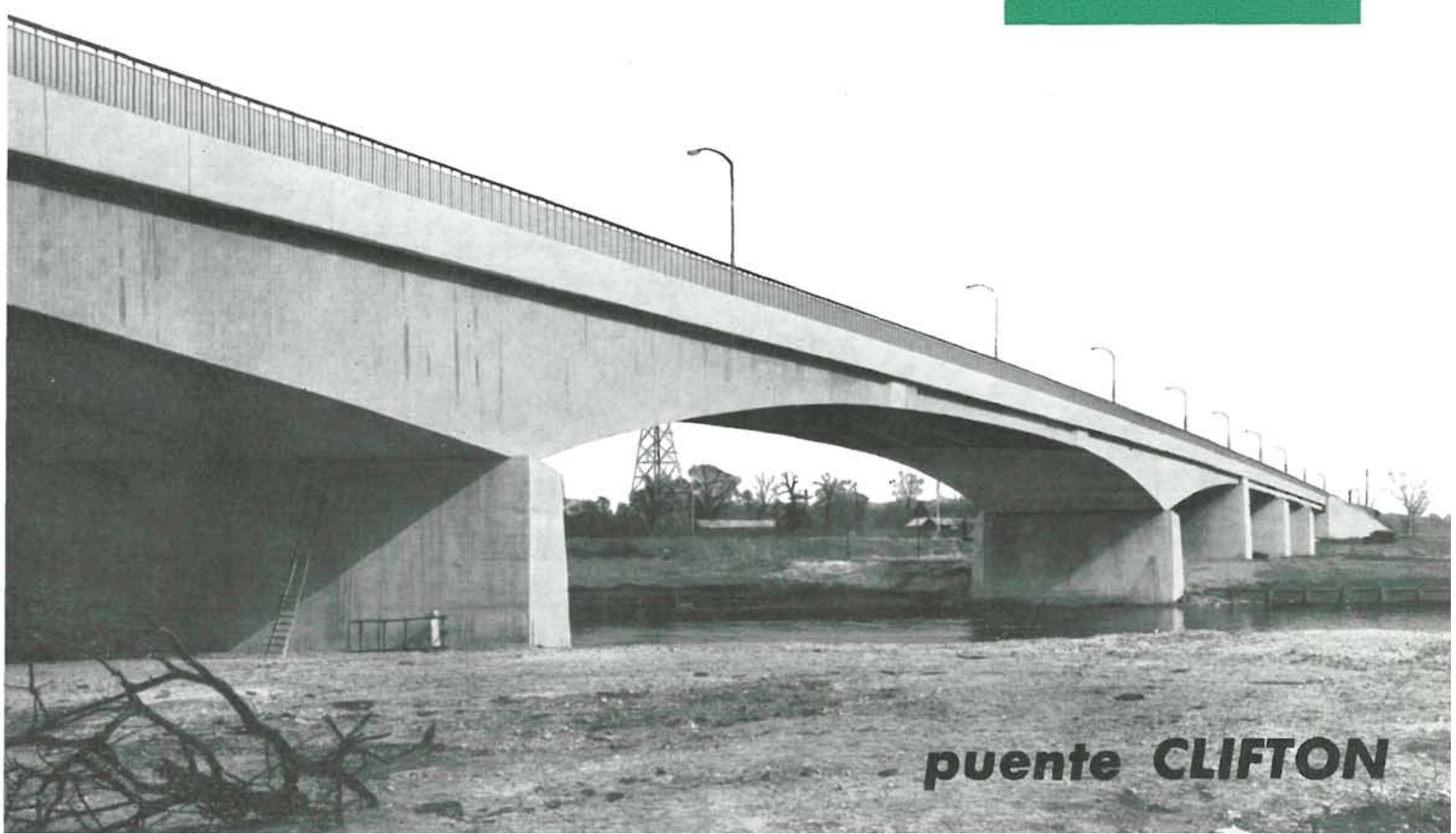


La carretera de los accesos tiene su plataforma sobre un terraplén de $9,15 \mathrm{~m}$ de cota, para evitar, en la medida de lo posible, las inundaciones de la calzada en los desbordamientos del río.

La parte más importante de la obra se concentra en el tramo sobre el cauce del río y los dos adyacentes a él. El tramo principal tiene $83,80 \mathrm{~m}$ de luz; y los dos laterales a él, 38. De tipo cantiléver, de hormigón pretensado, es el de mayor longitud en el país, actualmente, en su clase. En las márgenes, y con objeto de dar paso a las aguas en caso de avenidas, se han construído otras obras cuyos tramos son de menor importancia. Hacia el norte, y a unos $300 \mathrm{~m}$ del río, el terraplén que soporta la plataforma del camino se ha interrumpido tres veces con pontones de $12,20 \mathrm{~m}$ de luz. Al sur se han construído otras 3 estructuras que salvan luces de $27,50 \mathrm{~m}$. Estas obras complementarias forman parte de los accesos al puente de Clifton, que, por hallarse en una zona llana e inundable, ha requerido un desagüe. La longitud total de los 6 tramos del nuevo puente pretensado de Clifton es de $244 \mathrm{~m}$ : obra proyectada de acuerdo con las normas del Ministerio de Transportes que rigen actualmente.

El sistema Magnel-Blaton de pretensado se ha empleado en el puente propiamente y en los pontones construídos al sur, mientras que en las obras de fábrica, correspondientes al terraplén norte, ha sido adoptado el sistema LeeMcCall.

\section{Estudios preliminares}

Debido a una tendencia acentuada del río hacia avenidas periódicas, así como a la situación del puente en una parte llana, el régimen fluvial del Trent ha sido estudiado sobre un modelo reducido de la parte que al puente pudiera afectar. Como resultado de este estudio previo se llegó a la conclusión de la necesidad de apenas afectar el régimen hidráulico del río, dato que sirvió de base para el proyecto.

Hormigonando un soporte celular.

Principio de los obras.

Tramo adyacente al principal.
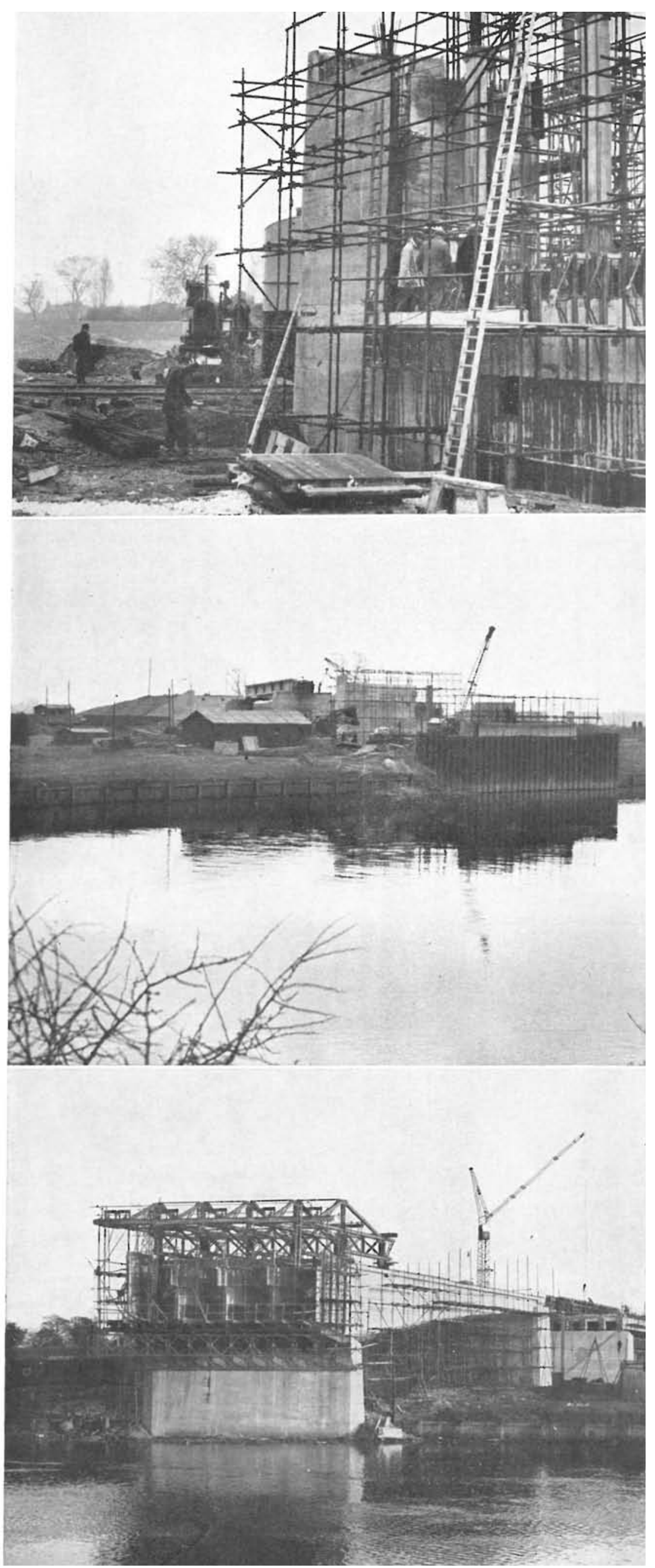

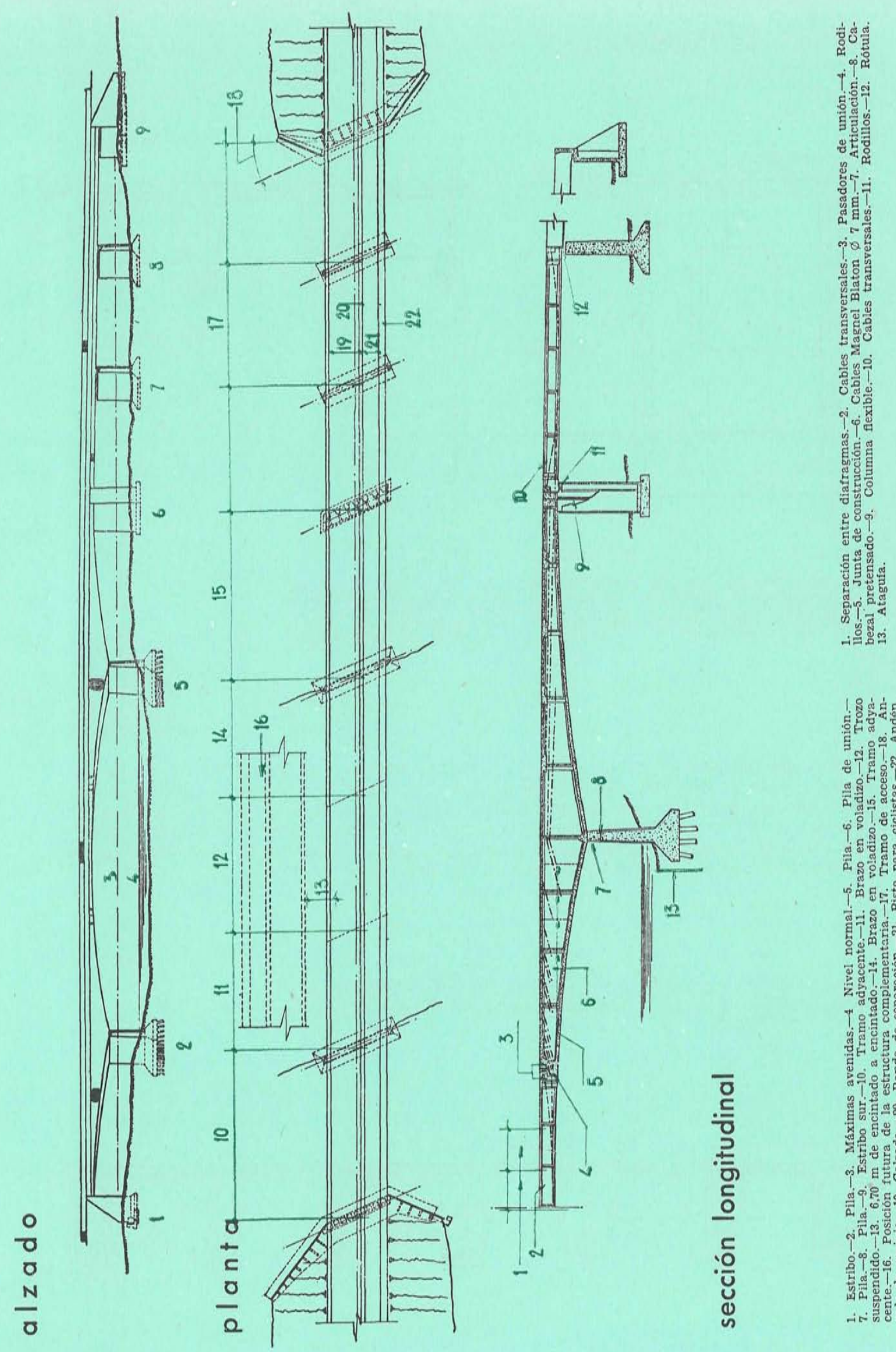

1 造密安

हैं क

ชา เุ็

通

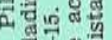

웅.

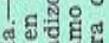

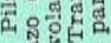

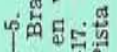

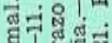

o m $^{\circ}$ ?

路我品

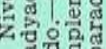

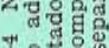

ㅂ. 랭

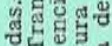

$\overline{0} \quad$ कां क्ष

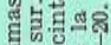

ํㅐㅇํำ के

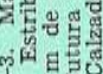

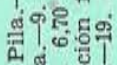

तिल

은

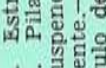




\section{detalle de tablero}

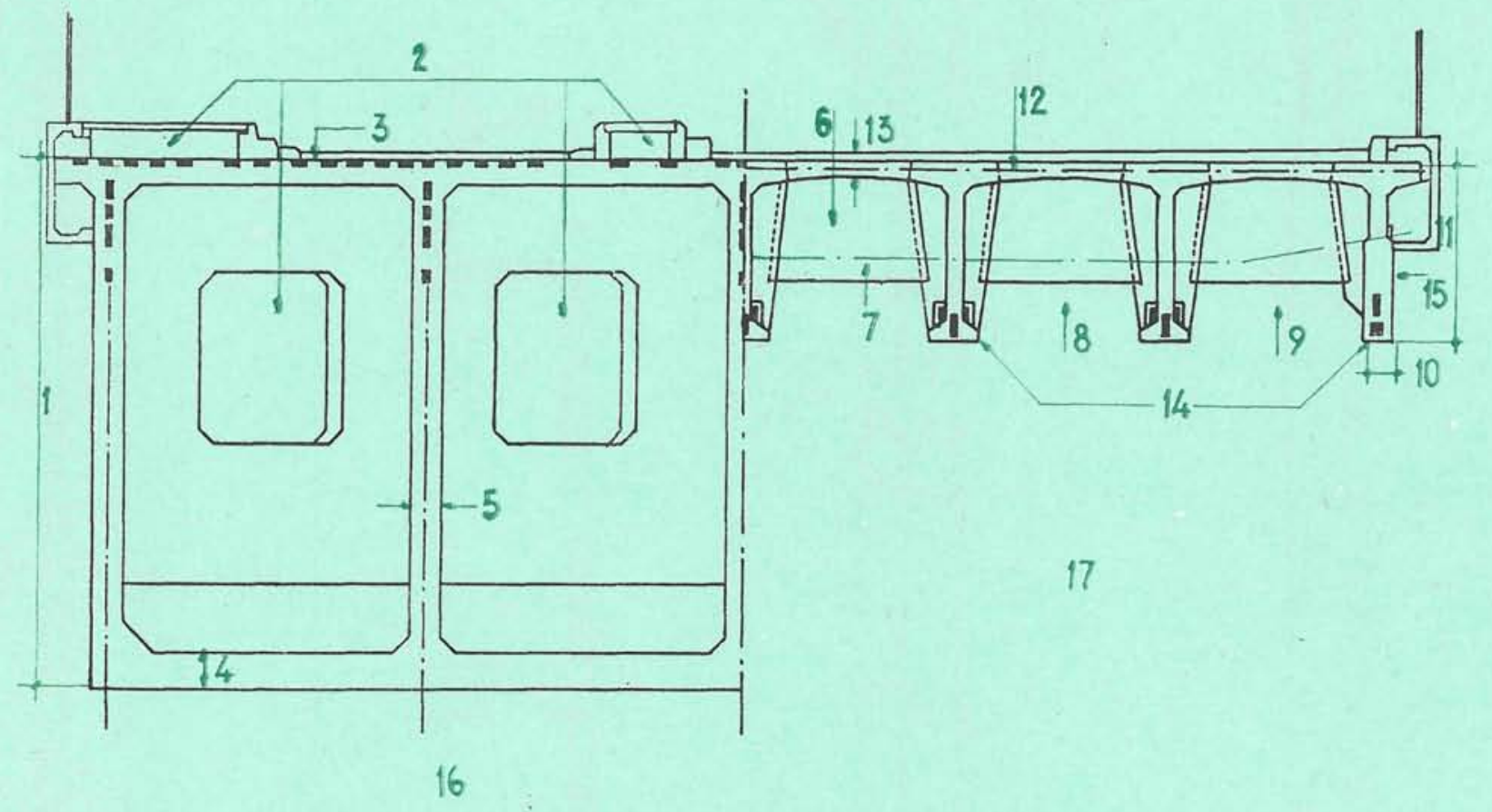

1. $5,50 \mathrm{~m}$ sobre pila; $2,00 \mathrm{~m}$ en la extremidad del brazo; $1,70 \mathrm{~m}$ sobre el apoyo del tramo adyacente. -2 . Huecos para canalización. -3 . Losa de $25 \mathrm{~cm} .-4$. Variable de 15 a $35 \mathrm{~cm} .-5$. Alma de $30 \mathrm{~cm} .-6$. Cables del diafragma.-7. Diafragma.-8. Cables.9. Cables, -10 . Cables,-11. $1,8 \mathrm{~m}$ en la clave.-12. Cables transversales.-13. Losa de $15 \mathrm{~cm} .-14$. Cables de $7 \mathrm{~mm} .-15$. Tímpano acanalado. -16 . Brazo en voladizo y tramo

Para determinar el efecto que una obra en esviaje pudiera inducir, se preparó un modelo a escala $1 / 50$ de la obra. Los cálculos teóricos de deformaciones en las secciones normales de la estructura se compararon con las deformaciones registradas en el modelo en esviaje. Se estudiaron una serie de cargas, deformaciones, esfuerzos de pretensado y efectos de esfuerzos cortantes, con objeto de establecer, variando la disposición de cargas, la posición de la reacción resultante en la extremidad de los tramos adyacentes al de mayor luz.

adyacente.-17. Vigas suspendidas.-18. Andén.-19. Pista para ciclistas.-20. Losa de $15 \mathrm{~cm} .-21,22,23$ y 24 . Cables. -25 . Juntas de construcción. -26 . Calzado. -27 . Capa de $15 \mathrm{~cm} .-21,22,23$ y 30 . Cables. -31 . En la clave 1,65.-32. Cables Magnel Blaton de $7 \mathrm{~mm} .-33$. Yontón sur.

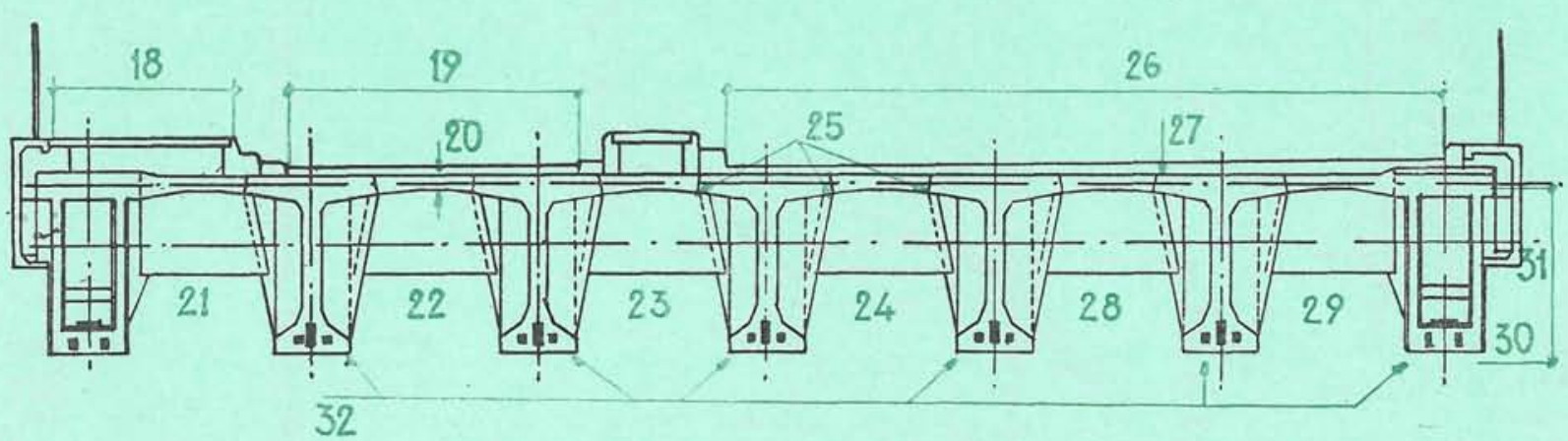




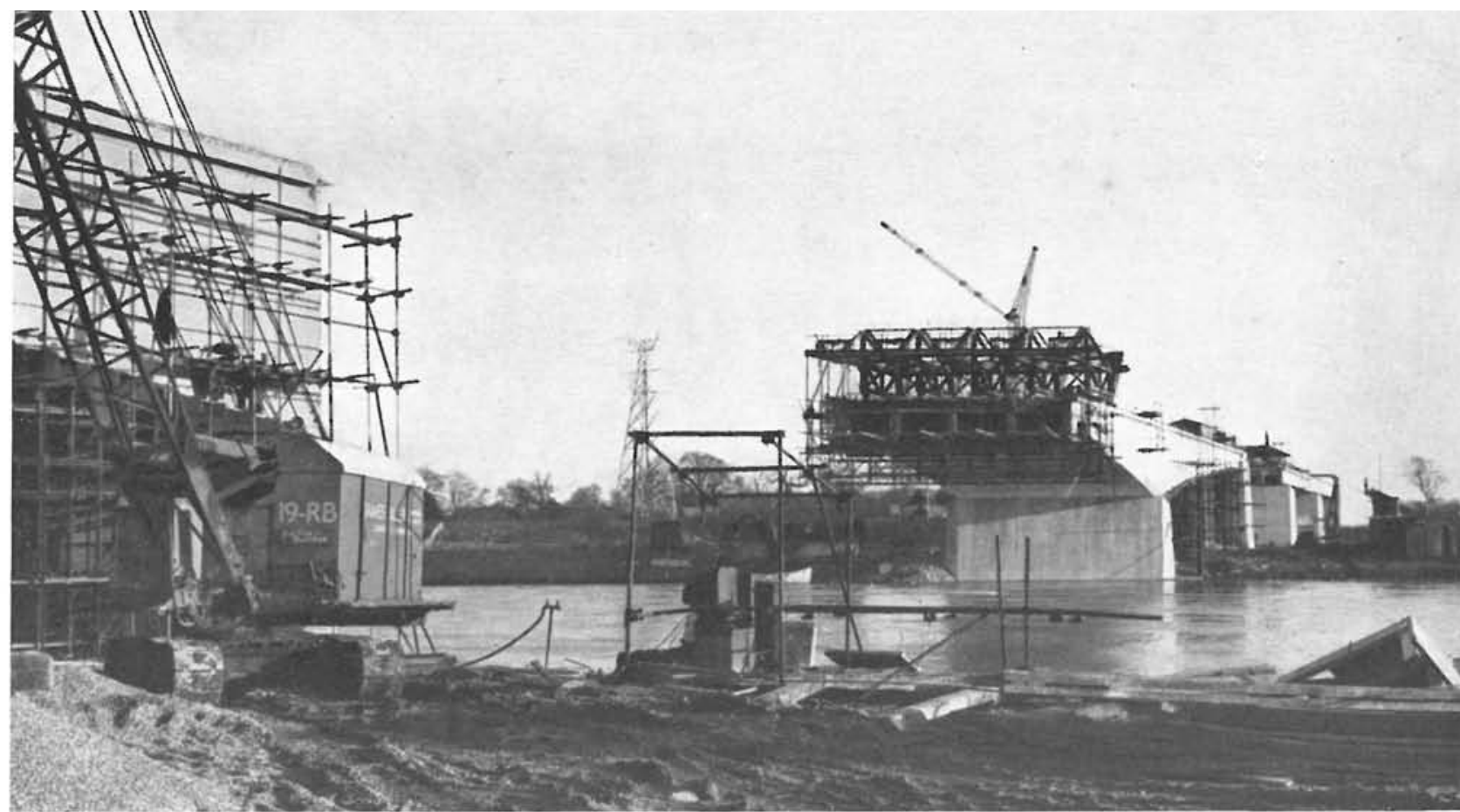

\section{Tramo principal}

El tramo principal se ha proyectado trabajando en dos brazos en voladizo equilibrado, de 26,70 metros de longitud, para cuyo contrapeso se contaba con los dos tramos adyacentes de $38 \mathrm{~m}$ de luz.

Los dos brazos volados se han unido con un trozo de vigas rectilíneas suspendidas, de $30,50 \mathrm{~m}$ de longitud. Tanto los brazos en voladizo como los dos tramos adyacentes, se han hormigonado "in situ"; pero las vigas del trozo de cierre se han prefabricado.

El estribo norte está formado por una estructura celular con alas de contrafuertes que acompañan el pie del terraplén.

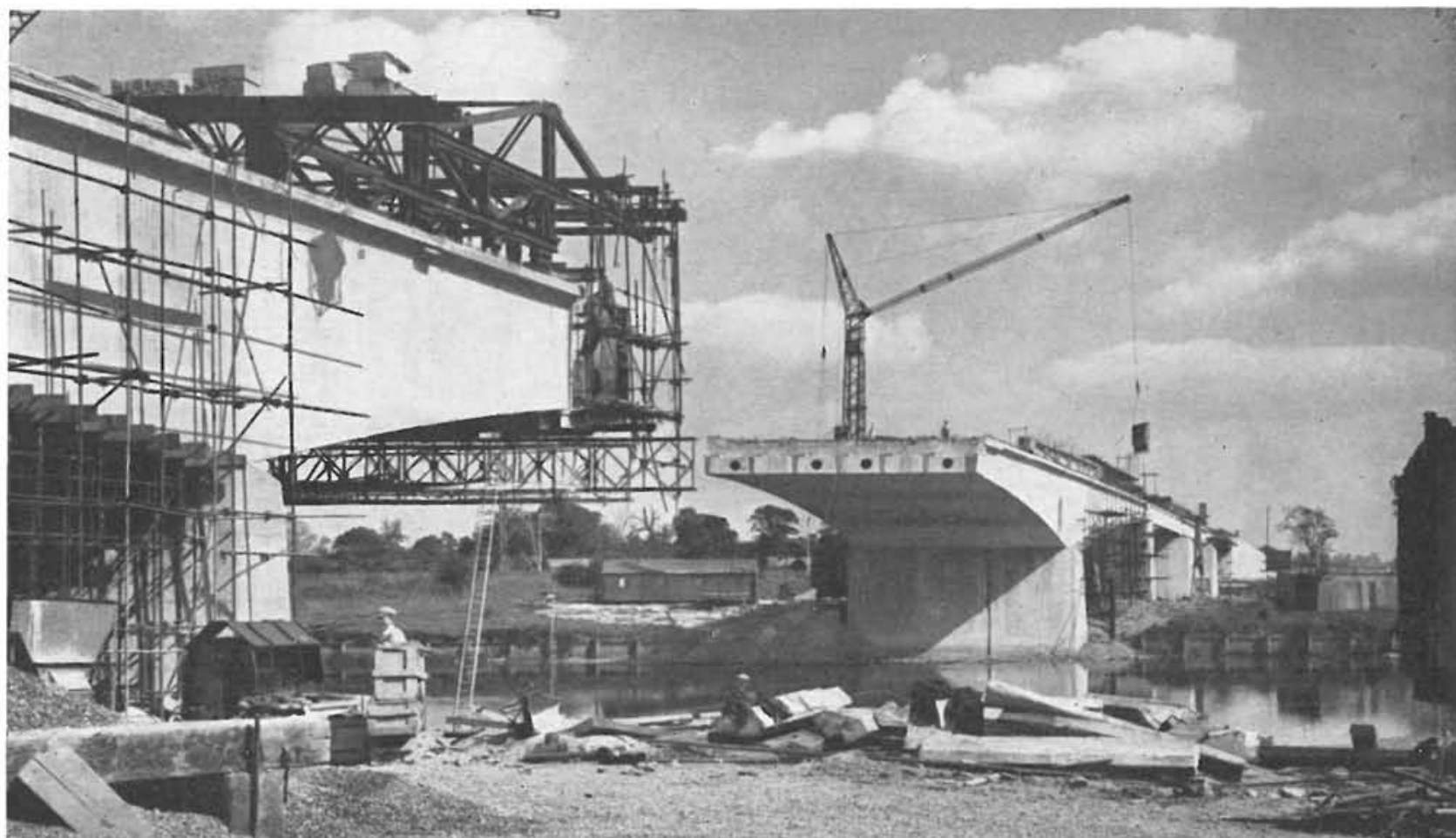



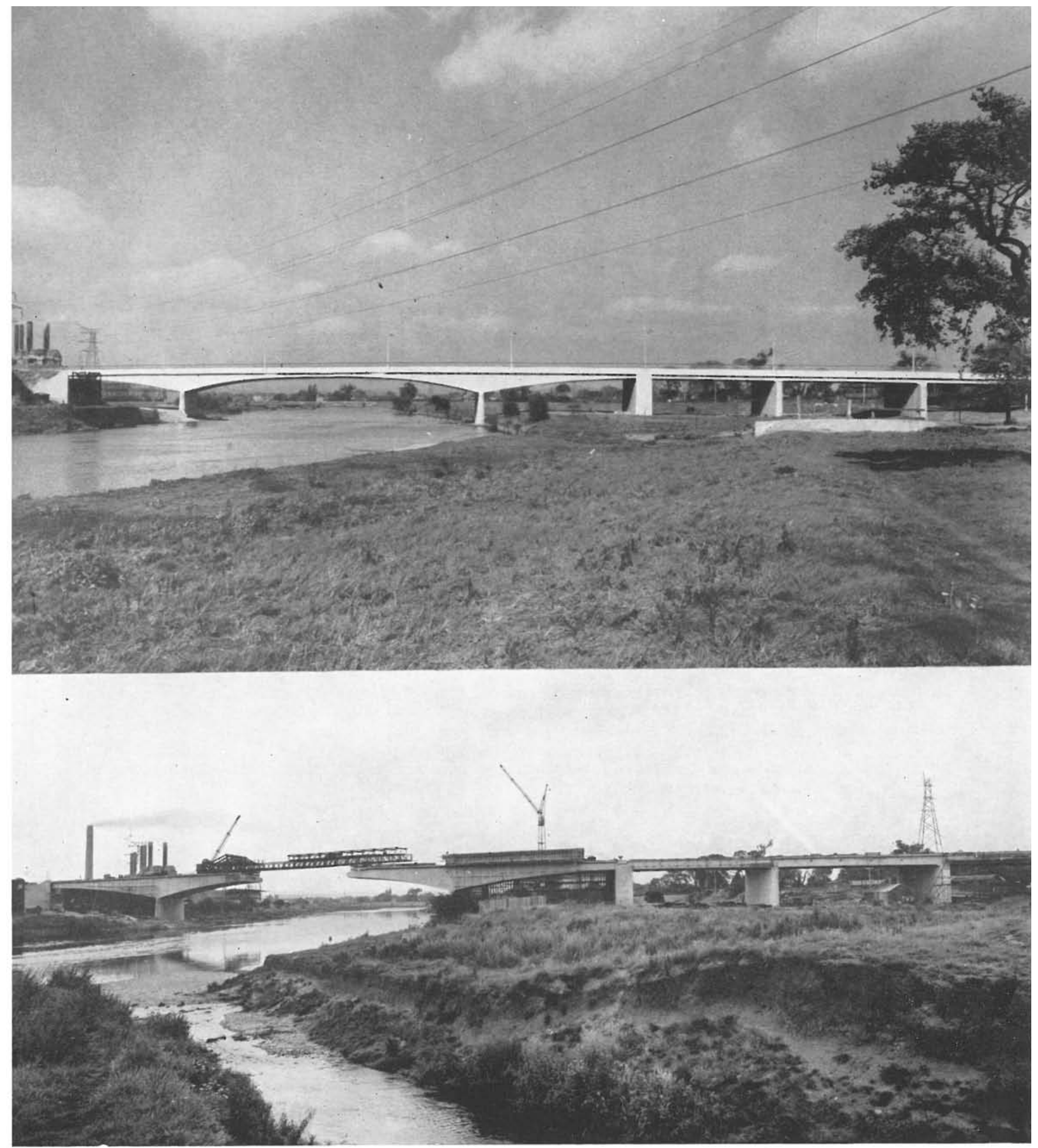

En la extremidad sur, el tramo adyacente se apoya sobre una pila celular que sirve de unión en el siguiente tramo. En el interior de este apoyo se aloja una columna flexible, pretensada, que soporta la extremidad del tramo adyacente. El tramo principal descansa sobre dos apoyos de hormigón armado que soportan los respectivos brazos equilibrados y en voladizo. Estos apoyos del tramo principal se apoyan sobre pilotes de hormigón. La construcción de estas pilas se ha realizado ayudándose de ataguías practicadas con tablestacas metálicas. En la parte superior de las pilas se han dispuesto, en cada una de ellas, un cabezal de hormigón pretensado sobre el que se apoyan las vigas que sostienen el tablero. 
Los dos brazos en voladizo se han construído formando viga cajón, con tabiques y diafragmas de $15 \mathrm{~cm}$ de espesor. Los tabiques longitudinales se han espaciado a $3 \mathrm{~m}$; mientras que los tabiques transversales o diafragmas, a $7,60 \mathrm{~m}$ en los tramos adyacentes y a $6,40 \mathrm{~m}$ en los brazos volados. La losa superior tiene $25 \mathrm{~cm}$ de espesor, variando de $35 \mathrm{~cm}$ sobre la pila a sólo 15 en la extremidad del tramo adyacente. Las canalizaciones para los servicios se han alojado en huecos formados debajo del tablero, a través de los diafragmas y a lo largo de las vigas prefabricadas en la parte central suspendida. Las canalizaciones para servicios más ligeros se han situado debajo de la losa del andén y de la banda que separa la pista para ciclistas y la calzada.

La construcción de los dos tramos adyacentes se realizó siguiendo los procedimientos ordinarios; pero la parte de brazos en voladizo, llevada a cabo posteriormente, por trozos de $3 \mathrm{~m}$ de longitud, se construyó con un encimbrado suspendido de la parte de brazo previamente construída, corriéndose cada vez que se terminaba y anclaba uno de estos trozos.

El pretensado de cada uno de los trozos y su anclaje se hizo utilizando los cables previstos para ellos, tesando convenientemente y anclando antes de proceder a la construcción del trozo siguiente, operación esta que se repitió cuantas veces fué necesario hasta la terminación del brazo. El pretensado se extendió a la losa del tablero y a los tabiques de las células que componen la viga cajón. Cuando era necesario, los cables de la losa se anclaban en el cuerpo de los tabiques dándoles una curvatura apropiada.

Las vigas del trozo central de cierre, de $30 \mathrm{~m}$ de longitud, de sección en forma de $\mathrm{T}$, de $2 \mathrm{~m}$ de canto en arranques y $1,80 \mathrm{~m}$ en la parte central, fueron prefabricadas en obra y se tesaron parcialmente para su colocación. Las vigas interiores tienen alas de $53 \mathrm{~cm}$ de anchura total en el fondo, mientras que las dos exteriores son de $28 \mathrm{~cm}$ de anchura total de alas, pero su alma es de mayor espesor. Estas vigas se apoyan sobre las extremidades de los brazos en voladizo, por el intermedio de rodillos en una extremidad y sobre rótulas en la otra. En los puntos de unión entre estas vigas y brazos se han colocado pasadores, de $28 \mathrm{~mm}$ de diámetro, para la transmisión de esfuerzos cortantes.

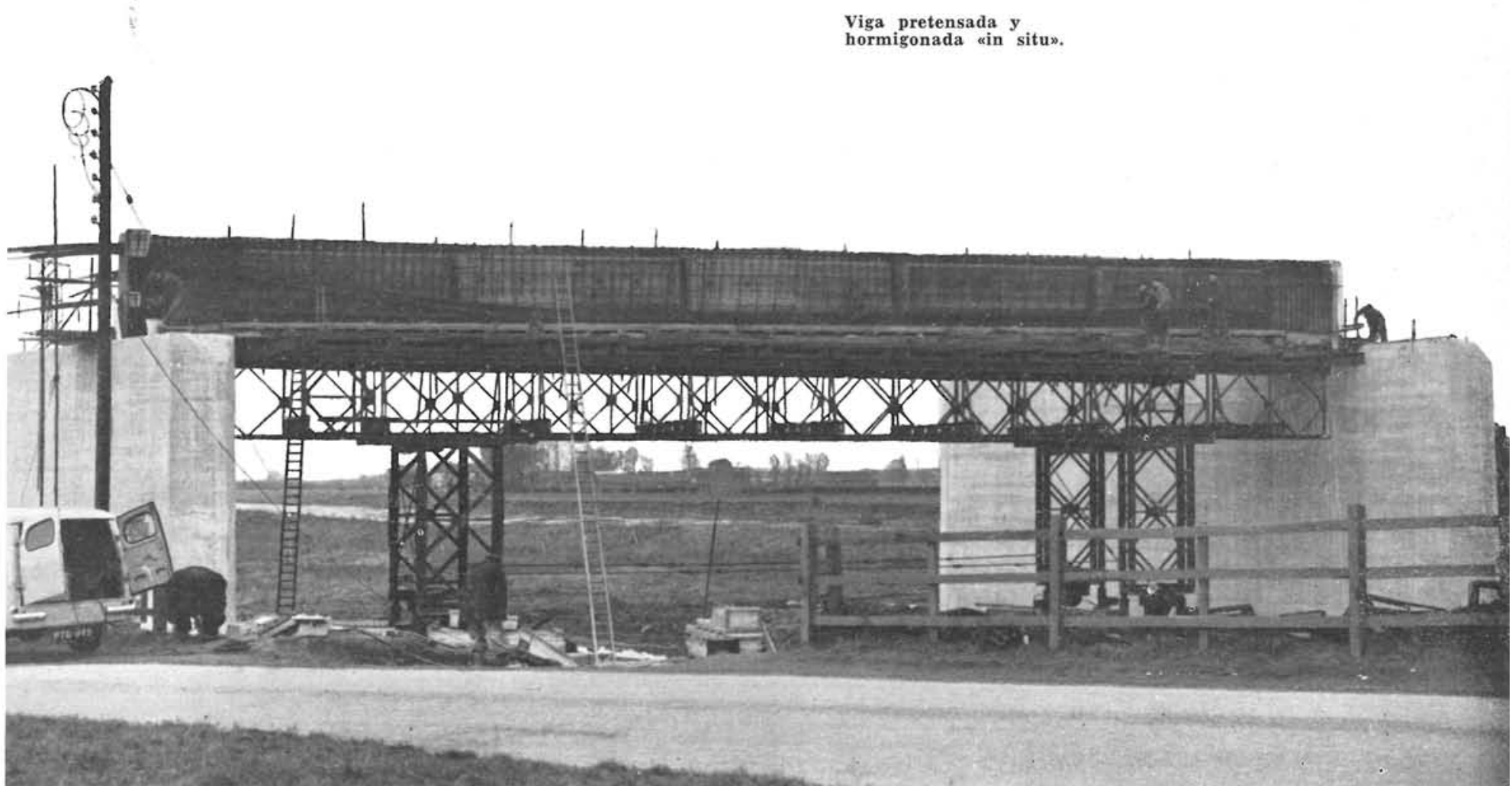




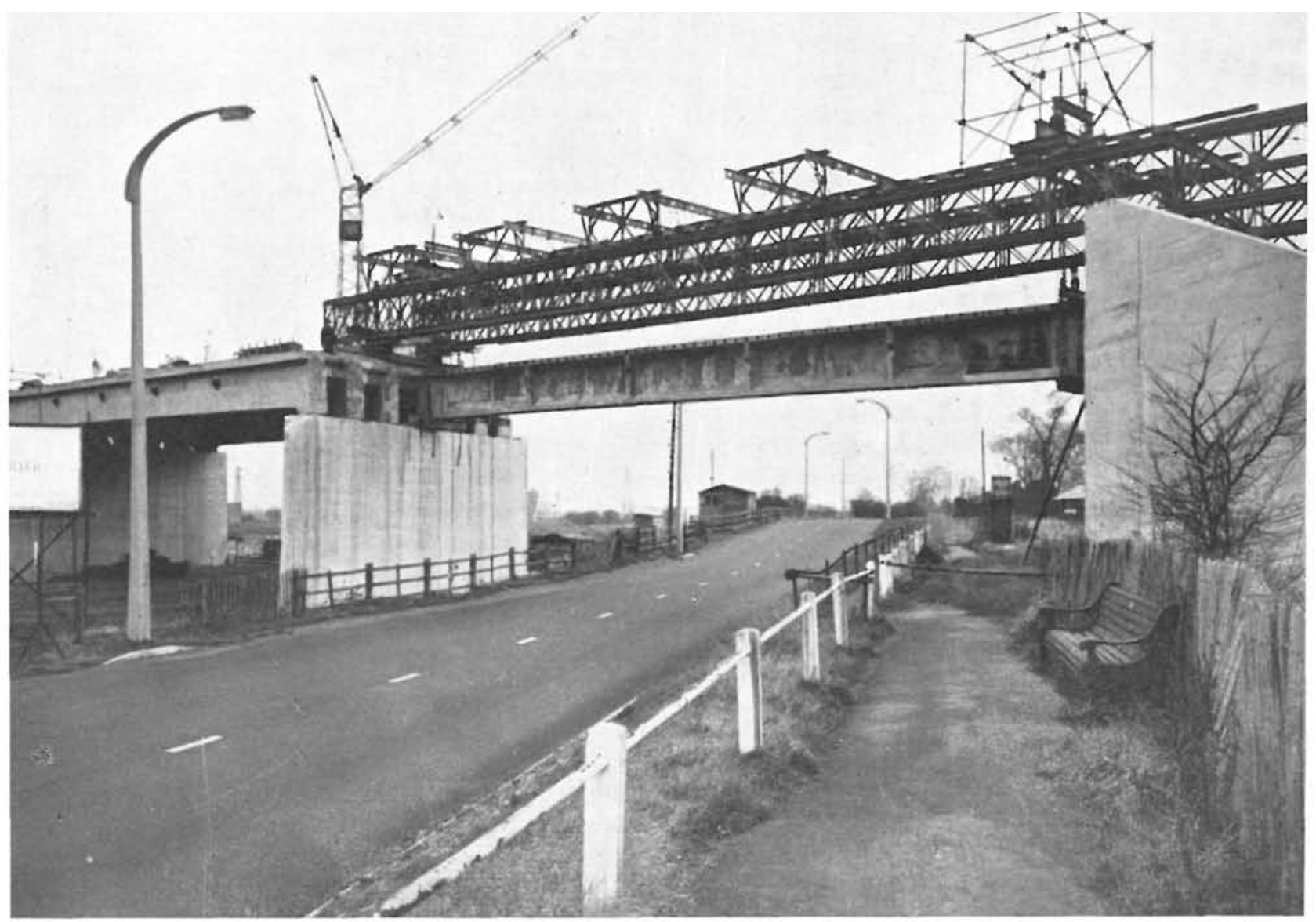

Tramo lateral para paso superior.

Después de haber colocado las vigas se procedió a hormigonar entre las alas con objeto de formar una losa monolítica de $15 \mathrm{~cm}$ de espesor mínimo. Al mismo tiempo que se hormigonaba, se iban formando los diafragmas transversales. Después de un buen fraguado del hormigón se procedió a pretensar transversalmente y a completar el tesado longitudinal.

En el pretensado transversal se ha utilizado alambre de $5 \mathrm{~mm}$ de diámetro en la losa, colocándolo según un trazado rectilíneo; pero en los diafragmas, el trazado es curvo. El pretensado longitudinal se ha realizado con armaduras de trazado curvo, dependiendo el número de armaduras de la carga de trabajo de cada viga.

\section{Obras complementarias}

Cada uno de los tres pontones, de $12,20 \mathrm{~m}$ de luz, de la margen norte, se componen de 16 vigas, de sección en forma de doble $\mathrm{T}$, prefabricadas y postesadas, siguiendo el procedimiento Lee-McCall antes de su colocación definitiva. El hueco entre vigas se cubrió con placas, las cuales sirvieron de encofrado para hormigonar la losa del tablero. Entre las vigas se hormigonaron elementos de rigidez. En el pretensado transversal se utilizaron tabiques constituídos por los elementos de rigidez para colocar las armaduras.

Cada una de las tres estructuras de la margen sur, que salvan luces de $27,50 \mathrm{~m}$, dispone de siete vigas de $1,70 \mathrm{~m}$ de canto. Las cinco vigas interiores son de sección en forma de doble $\mathrm{T}$; pero con objeto de conservar la forma adintelada de las vigas, las dos exteriores tienen una sección en forma de viga cajón. Las vigas interiores se han espaciado a $2,30 \mathrm{~m}$ y las exteriores a $2,00 \mathrm{~m}$. El pretensado se ha realizado siguiendo el sistema Magnel-Blaton, utilizando cables de $7 \mathrm{~mm}$ de diámetro.

Para dar cierta gracia arquitectónica a estas estructuras, entre las que se incluye la principal, se ha formado una imposta corrida, con áridos blancos, lisa, que contrasta con los tímpanos acanalados y grises del hormigón.

El Instituto de Ingenieros Civiles de Londres y la Cement and Concrete Association han contribuído a la redacción de este trabajo con su valiosa colaboración. 


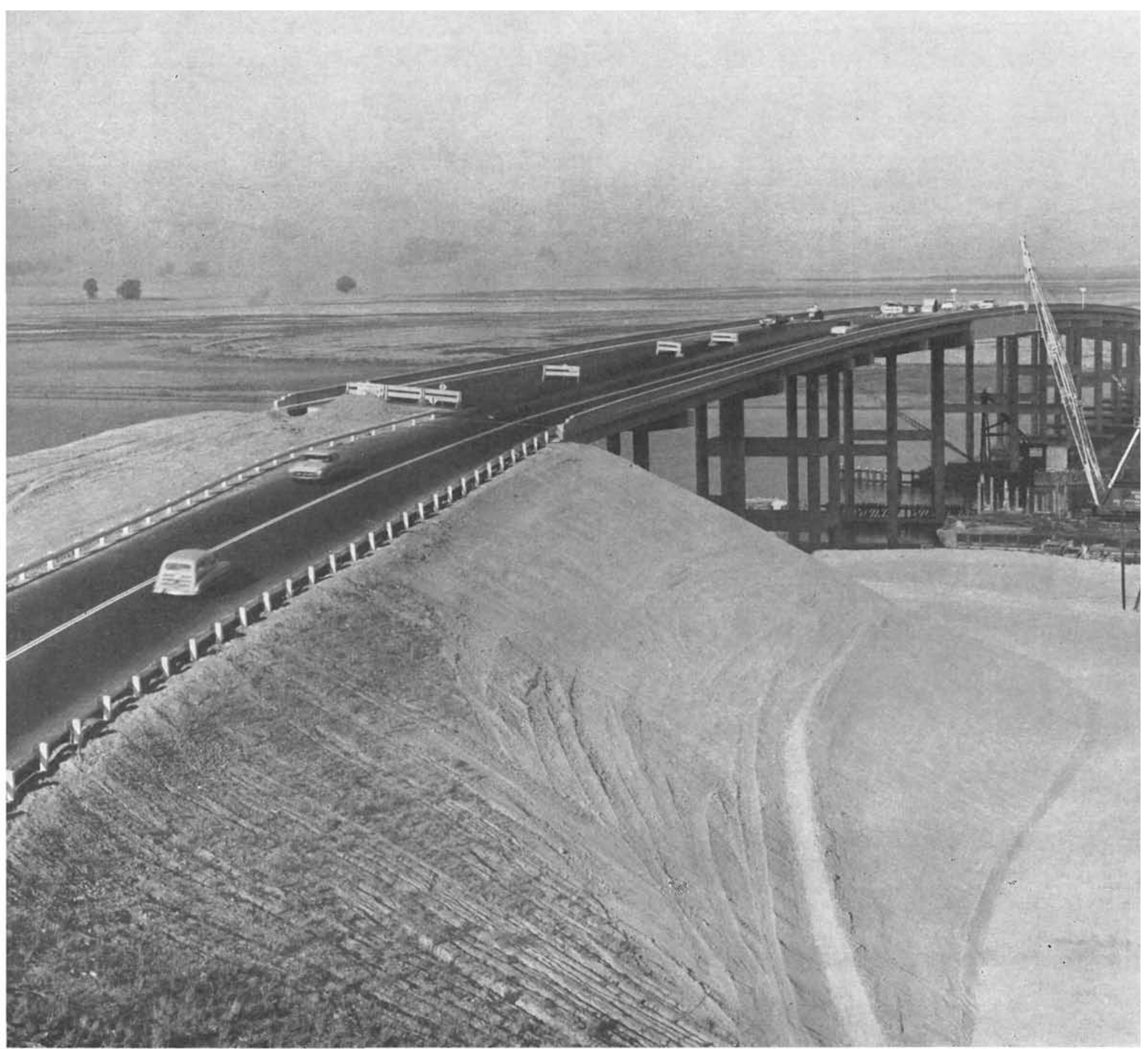

Anteriormente a la construcción del puente de Petaluma, California, que salva el río igualmente denominado, existía una estructura mixta, sometida ya a varias transformaciones, que tenía un tramo levadizo para dar paso a la navegación. Esta estructura había llegado ya a un estado de vejez que, junto con las nuevas mejoras de la carretera de Marín y Sonoma, transformada parcialmente en autopista, así como a el aumento continuo de tráfico, se pensó en construir un nuevo puente moderno que sustituyese al levadizo.

El canal exigía una altura libre mínima para dejar pasar la navegación por el río por una parte; pero como el terreno en que se halla el puente es fangoso, debido al efecto periódico de los mares, uno de los estribos tenía que crear dificultades para lograr la capacidad de sustentación que se necesitaba. Esto ha motivado que el puente se extienda en mayor longitud. Otro importante dato consistió en la necesidad de tener en cuenta los efectos sísmicos que se manifiestan con bastante frecuencia en esta zona.

La nueva estructura tiene $670 \mathrm{~m}$ de longitud y una altura media libre de $21 \mathrm{~m}$ en la zona del canal sometido a los efectos de la marea.

El puente consta de 29 tramos, de los que 24 corresponden a vigas rectas prefabricadas, de hormigón pretensado, de $1,35 \mathrm{~m}$ de canto y $24,50 \mathrm{~m}$ de longitud. 